\title{
The effects of labeling dimensional values on setting differences in shift performance of kindergarten children
}

\author{
JOAN H. CANTOR and CHARLES C. SPIKER \\ University of Iowa, Iowa City, Iowa 52242
}

\begin{abstract}
A reversal-shift paradigm was used to study the effects of dimensional labeling on the performance of 270 kindergarten children. Task 1 was a simultaneous discrimination problem in which form, size, and color were redundant relevant dimensions. In the shift task, one of the dimensions remained relevant, with reward contingencies reversed, and the other two dimensions were made irrelevant. In both tasks, the children named the stimulus object to be chosen, prior to choosing, in terms of one of the three sets of dimensional values. Shift performance was predicted to be superior in groups required to name relevant dimensional values in comparison with groups required to name irrelevant dimensional values. Moreover, shift performance was predicted to differ, within groups, for four different types of stimulus settings. Finally, differences in performance on the settings were predicted to be larger for the irrelevant-labeling groups than for the relevant-labeling groups. The results were found to be in good agreement with these predictions. The findings were discussed in relation to the subproblem analysis proposed by Tighe and Tighe (1972).
\end{abstract}

The discrimination-shift procedure has been widely used as a vehicle for determining the importance of dimension-specific responding in the discrimination learning process. The large body of literature that now exists involving the learning of reversal, nonreversal, optional, intradimensional, and extradimensional shifts has led many investigators to conclude that some type of dimensional transfer occurs in older children and adults under most conditions, and in younger children and subhumans under certain conditions. The postulated nature and function of the dimensional transfer varies considerably from one theoretical formulation to another, but typically involves some type of mediational process. The central question, however, is no longer whether or not some type of dimensional transfer occurs. The current questions are concerned with the conditions under which this type of transfer occurs and with the subsequent effects on discrimination learning.

The justification for the extensive use of the shift paradigms is that they provide a means of studying the conditions under which dimension-specific responses established in the original learning task transfer to the shift task. In all of these paradigms, instrumental as

Preparation of this report was supported by Grant BMS75-04334 from the National Science Foundation. The writers are indebted to Aletha Crowder, Adele Misurella, Paul Robinson, and Gerry Taylor for assistance in collecting and tabulating data. They are also grateful to the University of Iowa Graduate College for funds to purchase the computer time required for the data analysis. Deep appreciation is expressed to William Bleeker and David Cronin of the Iowa City Community School District for making subjects available, and to the principals and teachers in the Hoover, Twain, Lucas, Roosevelt, and Lemme Schools for their fine cooperation in many ways. well as dimensional transfer may occur, the amount depending upon the similarity of the stimuli used in the two tasks. Some investigators have preferred to study the joint effects of dimensional and instrumental transfer in shift learning (e.g., Kendler \& Kendler, 1968; Spiker \& Cantor, 1973). Others have attempted to eliminate, or at least control for, instrumental transfer in the hope of isolating the dimensional transfer effects (cf. Shepp \& Turrisi, 1966).

More recently, Tighe and Tighe (1972) have proposed a somewhat different strategy that may be most simply explicated within the context of an extradimensional shift. The subject first learns a simultaneous discrimination problem involving two nonspatial dimensions, say, size and brightness. In the first task, size may be relevant and brightness irrelevant. In the second, transfer task, brightness is made relevant and size is irrelevant. Given that the values of the two dimensions are not changed between tasks, it is necessary that one type of setting in the transfer task will have the reward contingency unchanged from the first task, whereas the other type of setting must have the reward contingency reversed. Tighe and Tighe have suggested that differential transfer performance on the two types of settings indicates that the settings are learned independently, and they have interpreted such performance as evidence that the subject learns the transfer problem more on the basis of object-reward relations than on the basis of an invariant dimension-reward relation. Conversely, they interpret the absence of differential setting performance as evidence that the subject has learned the transfer problem by means of the dimension-reward relation. In general, they have found that younger children and subhumans tend to show independent learning of the different 
settings (subproblems), whereas the setting differences are absent or markedly reduced in older children (Tighe, 1973; Tighe \& Tighe, 1972).

Subproblems are said to be learned independently if there is continued criterion-level performance on the unchanged setting paralleling a gradual increase from low to high performance on the changed setting. Independent learning, however, does not necessarily imply the learning of different content (e.g., different objectreward relations) for the two setting types. Although it is plausible to attribute differential setting performance to the learning of object-reward relations, there are other equally plausible interpretations. Indeed, Tighe (1973) appears to have recognized one such alternative explanation in his use of Spence's (1936) theory in computer simulations. He reported that the Spence theory, for a wide range of parametric values, predicted differential setting performance in extradimensional shifts. This theory assumes that exactly the same content (i.e., the accrual of habits and inhibitions to the values of the relevant and irrelevant dimensions) underlies mastery of the different settings. Although Tighe concluded that Spence's theory cannot account for certain other outcomes of subproblem analysis, the ability of the theory to predict independent subproblem learning indicates that independence might well be interpreted as the persistence of the invariant dimension-reward relation learned in the first task rather than as evidence for the learning of object-reward relations. In other words, differential subproblem performance may occur because the formerly relevant, now irrelevant dimension is at least partially controlling behavior during the early phase of transfer learning.

If the setting differences in the extradimensional shift are produced by the control of a formerly relevant, now irrelevant dimension, it should be possible to construct a transfer task in which several different setting types are present, with a different level of performance expected on each. Thus, if the initial task includes several redundant relevant dimensions, the transfer task might then have one of these dimensions relevant, with reward contingencies reversed, and with the other formerly relevant dimensions now irrelevant. The present authors (Spiker \& Cantor, 1973) have presented a quantitative theoretical analysis of such a paradigm, in which setting differences are predicted by a modified version of HullSpence theory (Spiker, 1970, 1971). In effect, this theory explains the second-task setting differences by means of the differential transfer of excitatory tendencies established to values of the relevant dimension(s) of Task 1 .

In the experiment reported here, setting differences in this paradigm were studied as a function of dimensional labeling in kindergarten children. In Task 1 , the children learned a simultaneous discrimination task having three relevant dimensions. In the reversal task, the correct cue was reversed on one of the three dimensions, and the remaining dimensions were made irrele- vant and variable within settings. The resulting transfer task was a problem in which performance differences among the setting types were predicted. In addition, the magnitude of these differences was expected to depend on the relevance of overt verbal labeling to the solution of the problem. Each child, prior to making a choice, verbalized the value chosen on one of the three dimensions throughout both learning tasks. Thus, one-third verbalized relevant dimensional values in the transfer task, and the remaining two-thirds verbalized irrelevant values.

In a less complex version of this paradigm having only one irrelevant dimension in the transfer task, Kendler and Kendler (1961) demonstrated that relevant labeling facilitates reversal learning, and irrelevant labeling interferes in relation to a no-label control condition. Silverman (1966) similarly demonstrated the facilitation effect with two irrelevant dimensions. However, performance was not analyzed separately for the various setting types in these studies, so that it is not known whether such setting differences occur and, if so, whether their magnitude depends on the relevance of the labels.

The present study had the threefold purpose of testing the following predictions from modified Hull-Spence theory (Spiker, 1970, 1971; Spiker \& Cantor, 1973). (1) Relevant dimensional labeling produces faster reversal learning than does irrelevant labeling; (2) performance differs for the various setting types in reversal learning; and (3) irrelevant labeling results in larger setting differences than does relevant labeling. These predictions will be explained in greater detail following a description of the tasks used.

The general nature of the experimental tasks can be explicated with reference to Figure 1. Task 1 is shown as a simultaneous discrimination problem with three relevant dimensions-size (large and small), form (square and circle), and color (green and blue). It is, of course, a two-setting problem, since the lateral reversal of the stimuli shown is presented on half the trials. In Task 2, for the counterbalancing condition illustrated, the form dimension is still relevant with reversed reward contingencies, and the other two dimensions are now

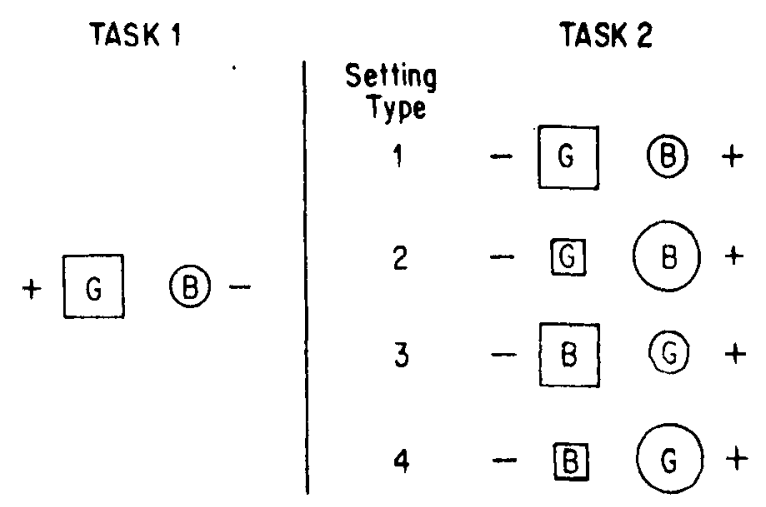

Figure 1. Illustration of the basic paradigm used in the present experiment. See text for explanation. 
irrelevant. The four types of settings when combined with their lateral reversals result in an eight-setting problem. On each trial, one group of kindergarten children was required to label the stimulus, prior to actual choice, with the name of its value on the dimension that was relevant in both tasks (form in the illustration); a second group was required to label with respect to one of the two dimensions that was relevant in Task 1 and irrelevant in Task 2 (color or size); and a third group was required to label with respect to the other such dimension. (The complete design included three replications, each having a different one of the three dimensions relevant in Task 2.)

The predictions for setting types in Task 2 can be best understood by comparing the correct stimulus compounds in the two tasks in Figure 1. Notice that the correct dimensional cues in Task 1 are square, large, and green. In Setting Type 1, the stimulus compounds are identical to those in Task 1, except for the reversal of reward contingencies. For these settings, transfer from Task 1 should therefore be negative on all three dimensions, since square, large, and green are all now incorrect. In Setting Type 2, however, the size cues have been interchanged from the first-task settings, so that large remains correct. Therefore, transfer to these settings should be positive with respect to size, though still negative with respect to form and color. Similarly, in Setting Type 3, green remains correct, so that transfer should be positive with respect to color and negative with respect to form and size. Finally, in Setting Type 4, large and green remain correct, so that transfer should be positive with respect to size and color, and negative with respect to form. To summarize, on the basis of instrumental transfer, performance in Task 2 should be poorest for Type 1 and best for Type 4. Types 2 and 3 might also be expected to differ if the size and color dimensions are differentially salient. For example, if the size difference was very salient relative to the color difference, performance should be better for Type 2 than for Type 3.

The specific equations from which these predictions can be rigorously derived have been presented elsewhere (Spiker \& Cantor, 1973), and only a brief summary of the basic tenets of the theory will be presented here. In this formulation, as in the Spence (1936) theory, the stimulus situation is analyzed into its dimensional components. Habit and inhibition are directly conditioned to components in reinforced and nonreinforced compounds, respectively. Both habit and inhibition are further assumed to generalize to similar components in all other compounds. According to the hypothesis of stimulus interaction (Spiker, 1970), the amount of generalization from one compound to another on a particular dimension depends not only on the similarity of cues on that dimension, but also on the similarity of cues on all other dimensions. Assuming, for simplicity, that drive is constant and equal to unity, excitatory tendency is equal to habit minus inhibition.
Finally, the total excitatory tendency for approaching a stimulus compound is obtained by summing over components in that compound. Performance is assumed to be a function of the difference in total excitatory tendency between the positive and negative compounds in a particular setting. According to the prediction equations derived for simultaneous discrimination learning, task difficulty is a decreasing function of both the number of relevant dimensions and the distinctiveness of the relevant cues. On the other hand, task difficulty is an increasing function of both the number of irrelevant dimensions and the distinctiveness of the irrelevant cues. Additional equations permit the prediction of performance in transfer tasks. The prediction of setting differences in the present study is based on differential transfer effects that depend on whether or not the reward contingencies have been changed from the first to the second task with respect to each of the dimensions. The verbal description of the predicted setting differences provided earlier completely parallels the quantitative predictions from the theory.

Within this theoretical framework, dimensional labeling or other types of cue-producing responses are assumed to result in the addition of a response-produced stimulus dimension that is either relevant or irrelevant, depending upon the dimension being labeled. Thus, children using relevant labels in Task 2 theoretically have an added relevant dimension, whereas those using irrelevant labels have an added irrelevant dimension. It is therefore expected that the relevant groups will learn Task 2 more rapidly than will the irrelevant groups.

With respect to the effects of type of labeling on differences between setting types, it can be predicted from the theory that setting differences should be greater with irrelevant labels than with relevant labels for one or both of the following reasons. (1) It will be recalled that setting differences are not dependent on the dimension that remains relevant in Task 2, since transfer is predicted to be negative on this dimension for all setting types. Rather, the setting differences depend on positive transfer with respect to one or both of the formerly relevant, now irrelevant dimensions in Task 2. Therefore, relevant labeling is not expected to enhance the setting differences, whereas labeling on a particular irrelevant dimension should enhance setting differences that are dependent on that dimension. This prediction can be best illustrated with reference to Setting Type 3, for which positive transfer is expected on the particular dimension labeled in each irrelevantlabel subgroup. It can be seen in Figure 1 that performance on Setting Type 3 is expected to be superior to that on Setting Type 1, because there is positive transfer on the color dimension in Type 3 and negative transfer on the color dimension in Type 1. This difference should be greater for subjects who are labeling the irrelevant color cues than for subjects who are labeling the relevant form cues. Furthermore, similar reasoning 
reveals that performance on Type 3 is predicted to be superior to that on Type 2 in the irrelevant-label groups. (2) Setting differences are theoretically dependent only on transfer from Task 1; therefore, they are expected to be largest at the beginning of Task 2 and to decrease over trials as Task 2 is learned. Since learning in Task 2 is expected to be faster in the relevant groups than in the irrelevant groups, setting differences should decrease faster over trials.

\section{METHOD}

\section{Apparatus}

The features of the stimuli that varied in the experiment are shown in Figure 1. The stimulus objects were eight laminated plastic blocks, $5.1 \mathrm{~cm}$ thick and varying in form (square vs. circle), color (blue vs. green), and size (large vs. small). The squares were $8.9 \mathrm{~cm}$ (large) and $5.4 \mathrm{~cm}$ (small) on a side, and the circles were $10.2 \mathrm{~cm}$ (large) and $7.6 \mathrm{~cm}$ (small) in diameter. Lifting the upper half of a block revealed a marble well inside, and a correct response was designated by the discovery of a marble placed in the well. Members of an identical set of eight blocks were substituted in a random fashion throughout training to discourage subjects from attending to incidental cues.

The blocks were presented either in the settings shown in Figure 1 or in the lateral reversals of these settings, with the reward contingencies appropriate for the particular counterbalancing condition. The apparatus, a modified Wisconsin General Testing Apparatus, was painted gray and consisted of a sliding wooden tray and a vertical partition separating the experimenter from the subject. The tray was retracted from the child's view while the experimenter placed and baited the blocks appropriately for the next trial. The blocks were placed on the tray $14.0 \mathrm{~cm}$ apart and were held in position with magnets. A trial was initiated by pushing the tray forward, exposing the blocks to the subject. After the subject had verbalized his choice on the appropriate dimension, he opened the block to look for the marble reward. The marbles were saved in a container placed next to the apparatus. An overhead lamp was used to provide constant illumination of the blocks.

\section{Design}

The details of the experimental design can be described with reference to Figure 1 , since the setting pairs were the same for all subjects. Every group received Task 1 with all three dimensions relevant, half having the task shown in the figure and the other half having the reward contingencies reversed. In Task 2, one-third of the subjects were given the settings illustrated, with form relevant and the appropriate reward contingencies for their counterbalancing condition. Another third were given the same stimulus settings, but with the reward contingencies arranged to make color relevant. The remaining third were given the same task except that size was made relevant.

During the learning of Task 1 , one-third of the subjects were required to say, prior to choice on each trial, whether the round or square block would contain the marble. Another third were required to say whether the blue or the green one would contain the marble, and the remaining third were required to say whether the large or the small one would have the marble. This practice was continued into Task 2, which was administered without interruption following Task 1. Basically, then, the design included three replications of the three conditions of verbalization, each replication involving a different relevant dimension in the shift task. Thus, in each replication, one group used relevant labels, and the remaining two groups used irrelevant labels in the shift task.

\section{Subjects}

The subjects were 270 kindergarten children (mean CA of $6-0)$ from the lowa City public school system. Thirty children were randomly assigned to each of the nine subgroups. Each child was brought individually to a mobile research laboratory for one 20 to $30 \mathrm{~min}$ experimental session. The data for 33 additional subjects were discarded, 25 for failure to learn Task 1 and 8 as a result of experimenter errors. The unusually large proportion of subjects who failed to meet criterion on the first task is probably the joint result of requiring the subject to name the stimulus objects prior to choice and having a relatively high performance criterion (seven of eight correct) for a total of only 16 trials.

\section{Procedure}

The first two trials of Task 1 also served as instructional trials. The child was told that two blocks would appear on each trial and that one of the blocks had a marble inside. He was asked to tell each time, before opening the block, which kind of block he thought the marble was in, using dimensional names appropriate for his group. If the subject chose the correct block, he was verbally rewarded and shown where to save his marbles. If he chose the incorrect block, the experimenter immediately pushed the tray back to prevent the subject from opening the other block and said, "No, it wasn't in the ___ one." Following Trial 2, which was conducted in the same manner as Trial 1, the experimenter said, "Now I'll go behind and stay there. There is a way to find the marble every time. After you know how to do it, be sure and get the marble every time." The experimenter continued to remind the subject to name the block chosen for several trials. Additional instructions were repeated as needed to maximize learning in Task 1 . All subjects received 16 noncorrection trials on Task 1 , and those who failed to achieve seven correct in the last eight trials were eliminated from the experiment. The 25 subjects who were eliminated by this criterion were distributed uniformly among the three verbalization conditions. A single sequence of settings was used in which each of the two settings appeared twice in every block of four trials.

Task 2 began immediately following the final trial in Task 1 , with no change in procedure. The setting presented on the first trial was one in which the reward contingency was reversed on all three dimensions (Setting Type 1), so that this trial provided all groups with information concerning the change in reinforcement. A minimum of 32 trials were administered, and subjects who failed to achieve a criterion of seven correct in each of two successive blocks of eight trials were given a maximum of 48 trials. The stimulus settings were administered in an order designed to produce chance performance for subjects using stimulus alternation or win-stick, lose-shift strategies on the irrelevant dimensions. The order was the same for all groups with respect to setting type. Within each successive block of eight trials, the eight settings each appeared once.

\section{RESULTS}

\section{Task 1}

For each subject, the proportion of correct responses was computed for each block of four trials in Task 1. These proportions were then entered into an analysis of variance in order to determine whether there were significant differences in performance resulting from random constitution of groups and/or the use of different dimensional labels. The analysis included Trial Blocks as a within-subject factor and, as between-subject factors, Dimension Labeled (form, size, or color) and 
Positive Compound (large green square or small blue circle). Only the main effect for Trial Blocks was significant $(p<.01)$, indicating that the different subgroups were rather well matched.

\section{Task 2}

In the shift task, the basic score was the proportion of correct responses for each setting type in each eighttrial block. Thus, each score was based on two trials, with the exception of the first trial block for Setting Type 1. It will be recalled that all subjects received this setting type on the first shift trial, constituting a complete reversal of reward contingencies on Task 1 stimuli with no forewarning. Since virtually all subjects made an error, as expected, including this trial would spuriously lower the first trial block mean for Setting Type 1 . Therefore, in this case, the trial block score was based on one rather than two observations. For subjects meeting criterion prior to the last trial block, the same scores were assigned for postcriterial blocks as were obtained on the criterial block.

The results of primary interest are presented in Figure 2. The four upper curves show the performance across trials of the combined relevant naming groups on each of the four setting types. The four lower curves depict the corresponding performance of the combined irrelevant groups. The superior performance of the relevant groups is readily apparent, with no overlap between the two sets of four curves. Large setting differences appear in the irrelevant groups, as predicted, with performance poorest on Type 1, intermediate on Types 2 and 3 , and best on Type 4 . In the relevant groups, performance appears to be superior on Type 4 in the early trials. The consistency of these findings for the three different shift problems may be seen in Table 1, which presents the subgroup means combined over trial blocks.

The shift-task proportions were entered into an analysis of variance, with Trial Blocks and Setting Type as within-subject factors and with Relevance of Labels (relevant vs. irrelevant) and Shift Problem (form, color, or size relevant) as between-subject factors. The analysis confirmed the trends observed in Figure 2, with significant main effects for both Relevance of Labels, $\mathrm{F}(1,264)=265.43$, error mean square $\left(\mathrm{MS}_{\mathrm{e}}\right)=.513$, and Setting Type, $F(3,792)=31.84, M_{e}=.108$. As predicted, the interaction of these factors was also significant, $F(3,792)=8.45, p<.0001, M_{e}=.108$. Setting Type also interacted significantly with Trial Blocks, reflecting a decrease in setting differences across trials. No other significant $(p<.05)$ interactions involving Setting Type were obtained.

Other significant effects included the main effect of Shift Problem $(p<.0001)$, primarily reflecting the fact that the problem with size relevant was easier than the other two. Shift Problem also interacted with Relevance of Labels $(p=.0001)$. It can be seen in the final column in Table 1 that this interaction reflects the somewhat

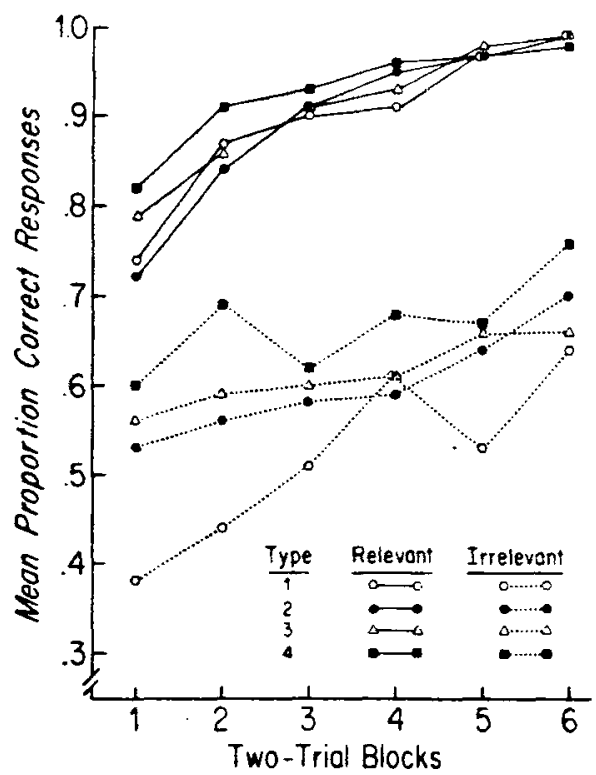

Figure 2. The mean proportions of correct responses for the combined relevant-label groups and the combined irrelevantlabel groups on each setting type.

smaller difference between relevant and irrelevant labeling in the group having size relevant. The only other significant effects were for Trial Blocks, Trial Blocks by Shift Problem, and Trial Blocks by Relevance of Labels.

Because of their theoretical importance, setting-type differences were examined in more detail in follow-up tests of the interaction of Setting Type by Relevance of Labels. The means combined over Trial Blocks and Shift Problems are presented at the bottom of Table 1 . For the combined relevant groups, the main effect of Setting Type was significant, $F(3,261)=3.16, p=.025$, $\mathrm{MS}_{\mathrm{e}}=.042$. Additional tests revealed that the source of this effect was the superiority of Setting Type 4 to Setting Types 1 and $2(p<.01)$. The main effect of Setting Type was also significant for the combined irrelevant groups, $F(3,531)=30.00, \quad p<.0001$, $\mathrm{MS}_{\mathrm{e}}=.141$. Additional tests showed Type 4 to be superior to Types 2 and $3(\mathrm{p}<.0001)$, which, though not differing from each other, were both superior to Type $1(\mathrm{p}<.001)$

Table 1

Means of Proportion Correct Responses for Each Setting Type in the Six Major Subgroups

\begin{tabular}{|c|c|c|c|c|c|c|}
\hline \multirow[b]{2}{*}{ Task } & \multirow[b]{2}{*}{ Labels } & \multicolumn{4}{|c|}{ Setting Type } & \multirow[b]{2}{*}{14} \\
\hline & & 1 & 2 & 3 & 4 & \\
\hline Form & $\begin{array}{l}\text { Relevant } \\
\text { Irrelevant }\end{array}$ & $\begin{array}{l}.90 \\
.45\end{array}$ & $\begin{array}{l}.93 \\
.54\end{array}$ & $\begin{array}{l}.89 \\
.55\end{array}$ & $\begin{array}{l}.92 \\
.63\end{array}$ & $\begin{array}{l}.91 \\
.54\end{array}$ \\
\hline Color & $\begin{array}{l}\text { Relevant } \\
\text { Irrelevant }\end{array}$ & $\begin{array}{l}.87 \\
.45\end{array}$ & $\begin{array}{l}.87 \\
.52\end{array}$ & $\begin{array}{l}.91 \\
.55\end{array}$ & $\begin{array}{l}.92 \\
.59\end{array}$ & $\begin{array}{l}.89 \\
.53\end{array}$ \\
\hline Size & $\begin{array}{l}\text { Relevant } \\
\text { Irrelevant }\end{array}$ & $\begin{array}{l}.91 \\
.66\end{array}$ & $\begin{array}{l}.89 \\
.74\end{array}$ & $\begin{array}{l}.93 \\
.74\end{array}$ & $\begin{array}{l}.95 \\
.79\end{array}$ & $\begin{array}{l}.92 \\
.73\end{array}$ \\
\hline $\begin{array}{l}\text { All } \\
\text { Tasks }\end{array}$ & $\begin{array}{l}\text { Relevant } \\
\text { Irrelevant } \\
R+I\end{array}$ & $\begin{array}{l}.90 \\
.52 \\
.64\end{array}$ & $\begin{array}{l}.90 \\
.60 \\
.70\end{array}$ & $\begin{array}{l}.91 \\
.61 \\
.71\end{array}$ & $\begin{array}{l}.93 \\
.67 \\
.76\end{array}$ & $\begin{array}{l}.91 \\
.60\end{array}$ \\
\hline
\end{tabular}




\section{DISCUSSION}

The results of the experiment show clearly that groups required to label the values of the relevant dimension performed better during the shift task than did groups required to label values of an irrelevant dimension. Furthermore, the superiority of the relevant labels held for all three replications, where each replication involved a different relevant dimension in the shift task. This finding is consistent with the prediction from modified Hull-Spence theory and also with earlier results using this paradigm (Kendler \& Kendler, 1961; Silverman, 1966). Relevant labeling has also been shown to facilitate reversal learning in related shift paradigms (e.g., Kendler, 1964, 1974; Kobayashi \& Cantor, 1974; Guldmann, Note 1).

It should be noted that overt labeling on an irrelevant dimension may have other effects on performance beyond the assumed addition of a set of irrelevant response-produced stimuli. Since the child is required to name the values on one dimension while learning to choose on the basis of another dimension, it might be argued that interference is produced in part by the complexity of his task in relation to that of a child who both labels and chooses on the basis of the relevant dimension. In addition, rehearsal might be more difficult for the child using irrelevant labels.

Primary interest here, however, was in the predicted setting differences and their interaction with type of labeling. The predicted ordering of the settings was demonstrated in the irrelevant labeling groups, except that Setting Types 2 and 3 did not differ significantly. In the relevant labeling groups, only Setting Type 4 proved superior to the other setting types. Theoretically, the latter differences were expected to be smaller and to decrease faster over trials. As seen in Figure 2, reversal learning was very rapid in the relevant groups, suggesting that the setting differences might be somewhat larger in a more difficult reversal task.

Within the present theoretical framework, the results are explained in terms of the hypothesized occurrence of cue-producing responses that add either a relevant or an irrelevant dimension to the task. Since the prediction of setting differences depends on whether irrelevant cues in a given setting type are expected to produce positive or negative transfer, irrelevant labels are expected to magnify the setting differences.

The results also appear to be consistent with those based on subproblem analyses reported by Tighe and Tighe (1972; Tighe, 1973). The paradigms on which they have focused (extradimensional and optional shifts) differ from the present paradigm in that one of the shift-task setting types (subproblems) is identical to a setting type in the original task, with no reversal of reward contingencies. Thus, one of their criteria for independent subproblem learning is near-perfect performance on the unchanged setting. In the present paradigm, on the other hand, the reward contingencies are reversed on at least one dimension (the relevant dimension) even though there may be no change on any of the irrelevant dimensions (Setting Type 4). Thus, in this paradigm, the magnitude of the setting differences, rather than the absolute performance level, should provide an index of the degree of independent subproblem learning. From this point of view, the present results are consistent with the Tighe and Tighe (1972) conclusion that dependence in subproblem learning is increased to the extent that there is dimensional control by the relevant dimension. Even the relatively young children $(\mathrm{CA}=6$ years) in this study were clearly brought under strong control of the relevant dimension by the relevant labeling. Similar dimensional control by the relevant dimension has been produced in a reversal task by perceptual pretraining prior to Task 1 (e.g., Tighe \& Tighe, 1969).

In the present study, irrelevant labeling produced considerably more subproblem independence, as predicted. This finding is consistent with the assumption that independence reflects, not the absence of dimensional control, but control by a formerly relevant dimension. The question still remains as to whether such subproblem independence would occur in the absence of overt labeling on the irrelevant dimension. According to the modified Hull-Spence theory, the setting differences are expected to occur in the absence of such labeling, and are merely magnified by irrelevant mediating responses. Some information on this point, however, may be found in the optional-shift literature. As Tighe and Tighe (1972) have noted, optional extradimensional shifts can be viewed as evidence of subproblem independence, since the test settings are identical to one of the original setting pairs and different from the pair of shift settings. If the child chooses during the test task in accordance with his Task 1 training, he is classified as having made an extradimensional shift. Optional shift data indicate that, in the absence of labeling or other special training, approximately $28 \%$ of 7-year-olds make extradimensional shifts (Kendler \& Kendler, 1970). Kobayashi and Cantor (1974) subsequently showed in a modified optional shift design that when 7-year-olds labeled the formerly irrelevant dimensional values during shift learning, $78 \%$ made extradimensional shifts. It should be noted that these children were a year older than those in the present study. Nevertheless, the results suggest that, in the absence of overt labeling, some children show independent subproblem leaming in the optional shift, whereas most show independence when they are required to label the formerly irrelevant cues.

The results of subproblem analysis, including the present results, pose serious difficulties for attention theories like that of Zeaman and House (1963), in which it is assumed that the correct choice response is dependent on the prior occurrence of an observing re. sponse to a single relevant dimension. Zeaman and House (1974) have more recently explained subproblem 
independence in terms of dimensional compounding. In applying the compounding hypothesis to the present situation, it might be assumed that the children learn Task 1 on the basis of color-form-size compounds. The prediction of setting differences would then depend on having some basis for determining the similarity of compounds. Let us assume, for purposes of discussion, that similarity among compounds is a function of the number of shared dimensional components. Then performance should be poorest in Setting Type 1, best in Type 4, and intermediate in Types 2 and 3. The present results are, of course, consistent with these predictions. However, it is not clear how the stimulus labeling would be expected to affect compounding. Since all of the children labeled on a single relevant dimension in Task 1, the question might be raised as to whether compounding would be expected to occur at all under these conditions. Furthermore, even if compounding does occur, it would seem that it should occur equally in all of the groups, and the differential effects of relevant and irrelevant labeling on setting differences remain to be explained.

In conclusion, the present results are consistent both with theoretical expectations and with results from related areas. The method of analyzing setting differences holds considerable promise for providing a fine-grained analysis of variables affecting dimensional control in discrimination learning.

\section{REFERENCE NOTE}

1. Guldmann, H. The effects of dimensional verbalization upon children's performance on reversal and extradimensional shift discrimination problems. Unpublished doctoral dissertation, University of lowa, 1972.

\section{REFERENCES}

Kendler, H. H., \& Kendler, T. S. Effect of verbalization on reversal shifts in children. Science, 1961, 134, 1619-1620.

Kendler, H. H., \& Kendler, T. S. Mediation and conceptual behavior. In J. T. Spence (Ed.), The psychology of learning and motivation (Vol. 2). New York: Academic Press, 1968. Pp. 197.244.

KENDLER, T. S. Verbalization and optional reversal shifts among kindergarten children. Journal of Verbal Learning and Verbal Behavior, 1964, 3, 428-436.
KENDLER. T. S. The effect of training and stimulus variables on the reversal-shift ontogeny. Journal of Experimental Child Psychology, 1974. 17, 87-106.

Kendler. T. S., \& Kendler, H. H. An ontogeny of optional shift behavior. Child Development, 1970, 41, 1-28.

Kobayashi, E. L., \& Cantor, J. H. The effects of dimensional naming upon children's performance in a modified optional shift problem. Memory \& Cognition, 1974, 2, 401-405.

Shepp. B. E., \& TuRrusi, F. D. Learning and transfer of mediating responses in discrimination learning. In $\mathbf{N}, \mathbf{R}$. Ellis (Ed.), International review of research in mental retardation (Vol. 2). New York: Academic Press, 1966. Pp. 86-120.

Silverman, I. W. Effect of verbalization on reversal shifts in children: Additional data. Journal of Experimental Child Psychology, 1966, 4, 1-8.

SPENCE, $K$. W. The nature of discrimination learning in animals. Psychological Review, 1936, 43, 427-449.

SPIKER, C. C. An extension of Hull-Spence discrimination learning theory. Psychological Review, 1970, 77, 496-515.

SPIKER, C. C. Application of Hull-Spence theory to the discrimination learning of children. In H. W. Reese (Ed.), Advances in child development and behavior (Vol. 6). New York: Academic Press, 1971. Pp. 99-152.

Spike R, C. C., \& Cantor, J. H. Applications of Hull-Spence theory to the transfer of discrimination learning in children. In $\mathrm{H}$. W. Reese (Ed.), Advances in child development and behavior (Vol. 8). New York: Academic Press, 1973. Pp. 223-288.

Tighe, L. S., \& Tighe, T. J. Transfer from perceptual pretraining as a function of number of task dimensions. Journal of Experimental Child Psychology, 1969, 8, 494-502.

TIGHE, T. I. Subproblem analysis of discrimination learning. In G. H. Bower (Ed.). The psychology of learning and motivation (Vol. 7). New York: Academic Press, 1973.

Tighe, T. J., \& Tighe, L. S. Stimulus control in children's learning. In A. D. Pick (Ed.), Minnesota symposium on child development (Vol. 6). Minneapolis: University of Minnesota Press, 1972. Pp. 128-157.

Zeaman, D., \& House, B. J. The role of attention in retardate discrimination learning. In N. R. Ellis (Ed.), Handbook of mental deficiency. New York: McGraw-Hill, 1963. Pp. 159-223.

ZEAMAN, D., \& HousE, B. J. Interpretations of developmental trends in discriminative transfer effects. In A. D. Pick (Ed.), Minnesota symposium on child development (Vol. 8). Minneapolis: University of Minnesota Press, 1974. Pp. 144-186.

(Received for publication September 5, 1975; revision accepted December 8,1975 .) 